\title{
Impresión tridimensional 3D en el área protésica: innovación $v s$ investigación
}

Desde la invención de la impresora de inyección de tinta en 1976, la tecnología continuó su evolución hacia la impresión con materiales. En 1984, Charles Hull inventó la estereolitografia en que, a partir de datos digitales, es posible crear modelos en 3D, usando un material acrílico conocido como fotopolímero. Desde entonces, la evolución de las tecnologías, asi como de los materiales disponibles, nos han permitido contar con una mayor diversidad de métodos para la impresión tridimensional (3D). Esto ha convocado el interés de diversos profesionales, ingenieros, diseñadores y a los mismos usuarios, a intervenir con nuevas ideas en diversas áreas de la salud ${ }^{1,2}$.

En el ámbito protésico, la manufactura aditiva es el modo de fabricación más usado para la impresión de partes y piezas en el diseño de prótesis. Las innovaciones se basan en trabajar modificaciones sobre dispositivos ofuncionalidades ya existentes, en confeccionar dispositivos protésicos impresos en 3D, usando materiales de bajo costo ${ }^{3}$. Esto promete dar mayor facilidad de acceso a los usuarios, a todo nivel, suponiendo un buen resultado funcional. Hoy, de hecho, están disponibles en la red varios diseños, que se obtienen gratuitamente 4 . Dicho esto, es obvio pensar que la posibilidad de contar en forma global y "democrática" con dispositivos protésicos de bajo costo y fácil ajuste, podría ocasionar un impacto clínico, social y económicamente significativo, lo cual aún no es fácil de evidenciar con la información disponible, al menos para los clínicos que trabajamos en esta área.

Es importante diferenciar la innovación de la investigación. La Organización para la Cooperación y Desarrollo Económico (OCDE), define la innovación como: "la introducción de un producto (bien o servicio) o de un proceso, nuevo o significativamente mejorado, o la introducción de un método de comercialización o de una organización nueva aplicada a las prácticas de negocio, o a la organización del trabajo o a las relaciones externas " 5 . La misma $O C D E$ se refiere a la investigación + desarrollo experimental $(I+D)$ como: "el trabajo creativo llevado a cabo de forma sistemática para incrementar el volumen de conocimientos, incluido el conocimiento del hombre, la cultura y la sociedad, y el uso de esos conocimientos para crear nuevas aplicaciones". El término I+D engloba tres actividades: investigación básica, investigación aplicada y desarrollo experimental ${ }^{6}$.

La investigación básica consiste en trabajos experimentales o teóricos que se emprenden principalmente para obtener nuevos conocimientos acerca de los fundamentos de los fenómenos y hechos observables, sin pensar en darles ninguna aplicación o utilización determinada.

La investigación aplicada consiste también en trabajos originales realizados para adquirir nuevos conocimientos; sin embargo, está dirigida fundamentalmente hacia un objetivo práctico especifico.

El desarrollo experimental consiste en trabajos sistemáticos que aprovechan los conocimientos existentes obtenidos de la investigación y/o la experiencia práctica, y está dirigido a la producción de nuevos materiales, productos o dispositivos; a la puesta en marcha de nuevos procesos, sistemas y servicios, o a la mejora sustancial de los ya existentes. 
De las definiciones, queda claro que la innovación se basa en el desarrollo experimental $y$ está orientada a la obtención de un producto, que puede ser comercializado, para lo cual, previamente se debe realizar un estudio de mercado y un plan de negocios. La investigación, ya sea básica o aplicada, está enfocada a la obtención de nuevos conocimientos teóricos o prácticos.

Por esto entonces el llamado es a estar atentos, desde la responsabilidad de nuestro quehacer, en identificar y señalar, qué es lo esencial, lo necesario a mantener del objeto o proceso que está siendo sujeto de una posible innovación por un equipo de desarrollo.

En este contexto, hoy el desafio para aquellos que trabajamos en Rehabilitación, es participar en éstas dos áreas al mismo tiempo: en el ámbito de la investigación, produciendo información cientifica con evidencias válidas, en ámbitos clínicos relevantes, con un adecuado sustento estadístico, y al mismo tiempo, integrarnos a los equipos que estén trabajando en innovación, junto a usuarios y otros profesionales o técnicos de diversas disciplinas, para el desarrollo y aplicación práctica y funcional de las ideas.

Finalmente, nuestra meta debiera ser asegurar que el resultado de una posible innovación, cualquiera sea su magnitud o alcance, se transforme en una verdadera solución, que otorgue respuesta a las necesidades y expectativas expresadas por los usuarios; tenemos el deber y la responsabilidad de cautelar el cumplimiento de aquellos aspectos relacionados con la calidad de vida en salud, apuntando a optimizar en nuestros pacientes su desempeño funcional, actividad y participación, favoreciendo que ellos se hagan cargo lo más posible de su propia vida.

Dra. Jacqueline Dote Fisiatra

Subdirectora Laboratorios órtesis y prótesis Institutos Teletón

\section{Referencias bibliográficas}

1. Rengier F, Mehndiratta A, von Tengg-Kobligk H, Zechmann CM, Unterhinninghofen R, Kauczor HU, et al. 3D printing based on imaging data: review of medical applications. Int J Comput Assist Radiol Surg. 2010; 5: 335-41.

2. Goldberg D. History of 3D printing: It's older than you are (That is, if you're under 30). [Internet] [citado el 1 de junio de 2019]. Disponible en: https://www.autodesk.com/redshift/history-of-3d-printing.

3. Ventola C. Lee. Medical applications for 3d printing: Current and Projected Uses. PT. 2014; 39 (10): 704-11.

4. Zúñiga J, Katsavelis D, Peck J, Stollberg J, Petrykowski M, Carson A, et al. Cyborg beast: a low-cost 3d-printed prosthetic hand for children with upper-limb Differences. BMC Research Notes 2015; 8: 10. DOI 10.1186/s13104-015-0971-9.

5. Organisation for Economic Co-operation and Development OECD/Eurostat (2005). Oslo Manual: Guidelines for collecting and interpreting innovation data. 3rd Ed. Paris: The Measurement of Scientific and Technological Activities, OECD Publishing; 2005. [Internet] [citado el 15 de junio de 2019]. Disponible en: http://www.oecd-ilibrary.org/science-and-technology/ oslo-manual_9789264013100-en.

6. OECD. Frascati Manual 2015. Guidelines for collecting and reporting data on research and experimental development. The Measurement of Scientific, Technological and Innovation Activities, OECD Publishing, Paris. [Internet] [citado el 15 de junio de 2019] DOI: http://dx.doi.org/10.1787/9789264239012-en. 